\title{
Galilean Quantum Field Theories and a Ghostless Lee Model*
}

\author{
Jean-Marc Lévy-LebLond**
}

\begin{abstract}
Galilean quantum field theories, i.e. kinematically consistent nonrelativistic quantum theories with an infinite number of degrees of freedom, are considered. These theories transcend the frame of ordinary quantum mechanics by allowing genuine particle production processes to be described. The general structure of such theories is discussed and contrasted with the typical structure of relativistic quantum field theories which they may serve to illustrate a contrario. Despite the mass superselection rule, and due to the weakening of local commutativity conditions, galilean quantum field theories are much less constrained than relativistic ones. The CPT and spin-and-statistics theorems do not hold here, neither does Haag's theorem.

Second-quantized quantum mechanics, some many-body theories (such as the polaron model) and static models are briefly examined, giving simple examples and counterexamples of the general properties asserted.

A Lee model with complete nonrelativistic kinematics is studied and shown to give a consistent non-trivial example of a galilean quantum field theory. In this "GaliLee" model, while all the desirable features of the usual Lee model remain, the ghost problem disappears and the local coupling limit gives meaningful expressions for the physical quantities. The $(\mathrm{V} \leftrightarrow \mathrm{N} \theta)$ sector is solved for the physical $\mathrm{V}$. particle whose renormalization constant is finite for local coupling, and for the $\mathrm{N}-\theta$ scattering amplitude, which obeys an exact effective range formula in the same local limit. The elementarity of the $V$-particle is discussed in relation with the $Z=0$ rule and Levinson's theorem which is found wanting. The case of an unstable $\mathrm{V}$-particle is also considered, and leads, for local coupling, to an exact Breit-Wigner formula for the $\mathrm{N}-\theta$ scattering cross-section.
\end{abstract}

\section{Introduction}

In this paper, we wish to discuss and illustrate the concept of galilean quantum field theories, that is consistent nonrelativistic quantum field theories, in agreement with the galilean principle of relativity.

There are, in our opinion, several reasons for such an investigation:

- it is interesting to know if the framework of nonrelativistic quantum theory is limited to the usual quantum mechanics, dealing with fixed numbers of particles, or if it can be enlarged to describe genuine field theories, allowing particle production processes, which usually only appear in relativistic situations. We will show that the second alternative holds.

* Revised Version. September 1966.

** Permanent address: Physique Théorique, Faculté des Sciences, 06-Nice, France. 
- such theories may be used to devise models of quantum fields which, while being widely different from relativistic fields, nevertheless behave in a kinematically consistent way, at the nonrelativistic level (for instance, incorporating recoil effects). We will submit to such a galilean treatment the Lee model, thereby improving, we do think, its properties.

- galilean quantum field theories may be used as a pedagogical tool to emphasize the specific properties of relativistic quantum field theories. We will try to compare them in such a way as to exhibit the huge differences between the two types of theories.

- as to the relation of such considerations to actual physical situations, let us note that the simplest example of a galilean quantum field theory is the second-quantized form of ordinary nonrelativistic quantum mechanics, which forms the basis of all modern treatments of many-body problems. More recently, quantum field-theoretic methods have been successfully used to treat low-energy nuclear reactions between light nuclei, by means of nonrelativistic Feynman diagrams [1]. This is a more sophisticated example since in these treatments each nucleus is considered as "elementary", so that the number of particles may vary in certain processes. It thus seems worthwhile to investigate the conceptual framework of such theories.

Different authors have led related investigations with, however, different point of views. DRESDEN and KAHN [2] have considered field theories with "persistent one-particle states", that is essentially theories without vacuam polarization. They only require euclidean invariance, explicitly disregarding galilean invariance, though they make some relevant comments on the subject ${ }^{1}$. REDMOND and URETsky [3] have studied the asymptotic condition (in-out formalism) in the particular case of second-quantized quantum mechanics. HEPP [4] has discussed the asymptotic behavior of more general galilean quantum field theories. ScHweBer [5] has shown how the nonrelativistic Bethe-Salpeter equation reduces to the Schrödinger equation, by using galilean invariance ${ }^{2}$.

The first section of this paper is devoted to a general discussion of galilean quantum field theories from an abstract point of view.

In the second section we briefly discuss if and how our considerations can be applied to the cases of second-quantized quantum mechanics, the polaron model and static models.

The third section is devoted to a more detailed presentation of a nontrivial consistent example of a galilean quantum field theory, namely a kinematically modified Lee model.

1 See especially in [2], the introduction (pp. 403 and 405) and section 5b.

2 The non-relativistic Bethe-Salpeter equation, incorporating galilean invariance, has also been considered recently by KRAMER and MEETz [6]. 


\section{Generalities on Galilean quantum field theories}

In order to study the general structure of a galilean quantum field theory, we shall list the main postulates to be obeyed by such a theory and comment upon their implications. We will make no attempts to mathematical rigor, nor to completeness, but we will try to emphasize the physical consequences of the postulates in order to make clear the profound differences between a galilean and a relativistic quantum field theory ${ }^{3}$.

Postulate 1 (Hilbert space). The states of the systems to be studied are represented by rays in a separable Hilbert space and the dynamical variables by operators in this Hilbert space, with the usual rules for computations of probabilities, etc. This is the ordinary linear structure of any quantum theory (superposition principle).

The only comment to be made here is that, corresponding to the existence of superselection rules [8] (see also [7]), the Hilbert space may be decomposed into a direct sum (or integral) of so-called coherent subspaces, mutually orthogonal and such that the superposition principle does not hold for vectors belonging to different subspaces. This restriction will soon be seen to be of fundamental importance in the present case.

Postulate 2 (Galilean invariance). Requiring the theory to be invariant under general galilean transformations, we are led to postulate, simply adapting to the present case the fundamental analysis by WIGNER of relativistic invariance in quantum theory [9] (see also [7]):

There exists a unitary projective (i.e. up to a factor) representation of the Galilei group $G$ in the Hilbert space $\mathscr{H}$ of the system ${ }^{4}$.

Let us analyze more precisely this postulate. Let

$$
g=(b, \mathbf{a}, \mathbf{v}, R)
$$

be a general Galilean transformation with $b$ a time translation, a a space translation, $\mathbf{v}$ a pure Galilean transformation and $R$ a three-dimensional rotation. We denote by $\mathfrak{U}(g)$ the unitary projective representation of $G$ in $\mathscr{H}$. To this representation corresponds a representation of the Lie algebra $\mathscr{G}$ of the Galilei group by hermitian operators in $\mathscr{H}$. More precisely, since we deal with a projective representation, we obtain a representation of a central extension of $\mathscr{G}$ by a one-dimensional Lie algebra [11], [12], [14]. Concretely, let us call $(H, \mathbf{P}, \mathbf{K}, \mathbf{J}, M)$ respectively, the infinitesimal generators of the time-translations, space-translations,

${ }^{3}$ We will have many occasions of referring to general results of relativistic quantum field theory and will consistently refer the reader to the book by STREATER and WIGHTMAN [7], which contains the references to the original works on the subject.

${ }^{4}$ General discussions of the Galilei group and galilean invariance in quantum physics may be found in references [10], [11], [12], [13], [14]. 
pure Galilean transformations, rotations and the one-parameter phase group. The following relations define the extended Lie algebra $\mathscr{G}$ :

$$
\begin{gathered}
{\left[J_{i}, J_{j}\right]=\varepsilon_{i j k} J_{k} \quad\left[J_{i}, K_{j}\right]=i \varepsilon_{i j k} K_{k} \quad\left[J_{i}, P_{j}\right]=i \varepsilon_{i j k} P_{k}} \\
{\left[K_{i}, H\right]=i P_{i} \quad\left[K_{i}, P_{j}\right]=i \delta_{i j} M} \\
{\left[J_{i}, H\right]=\left[K_{i}, K_{j}\right]=\left[P_{i}, P_{j}\right]=\left[P_{j}, H\right]} \\
=\left[J_{i}, M\right]=\left[K_{i}, M\right]=\left[P_{i}, M\right]=[H, M]=0 .
\end{gathered}
$$

As to the physical interpretation of these operators, it is clear that $H$ is the Hamiltonian of the system, $\mathbf{P}$ its momentum and $\mathbf{J}$ its angular momentum. $\mathbf{K}$ is related to the center-of-mass position operator. Most important is the fact that $M$ has to be recognized as the mass operator. We now are in a position to draw the following conclusions:

a) role of the mass (Bargmann's superselection rule). The mass appears as a central element of the extended Lie algebra $\mathscr{G}$, that is, it commutes with all the other elements, in particular with the Hamiltonian $H$. It is thus a conserved quantity. It is even a "super-conserved" quantity, giving rise to a superselection rule [11], [14]. This is due to the fact that the extension $\mathscr{G}$ is nontrivial so that a physically trivial transformation may result in a definite modification of the phase of the state vector, depending on the mass of the system. In order not to alter the physical properties of the system by such a transformation, we must forbid any superposition of states with different masses, thus obtaining Bargmann's superselection rule. It has the effect of breaking the Hilbert space $\mathscr{H}$ into mutually incoherent eigenspaces of the mass operator $M$. The consequences of this superselection rule are rather far-reaching with respect to the possible types of particle reactions allowed by galilean invariance. Suppose, for instance, that we treat a theory with only one kind of particle, with definite mass $m$. In this case, conservation of the mass implies conservation of the number of particles, thus forbidding any type of production process ${ }^{1}$. Since we are specially interested in obtaining galilean quantum field theories exhibiting such production processes, we will have to include in these theories several kinds of particles with mass values chosen in agreement with the mass conservation law. This role of the mass in galilean quantum field theories is quite characteristic and introduces on the possible theories restrictions which do not exist in relativistic quantum field theories. However, among all the differences between the galilean and the relativistic cases, this one only imposes constraints stronger in the galilean case. All the other differences, as will be seen, permit galilean theories to escape some of the most severe limitations met by relativistic theories.

b) position of the Hamiltonian (introduction of interaction). It is a characteristic feature of the Galilei group, as compared to the Poincaré 
group, that its derived group is a proper (invariant) subgroup, not containing the time-translations. This is easily seen on its Lie algebra (2), where it means that the derived Lie algebra $\tilde{\mathscr{G}}^{\prime}=[\tilde{\mathscr{G}}, \tilde{\mathscr{G}}]$ does not contain the generator of time-translations. In other words, the Hamiltonian $H$ never appears "on the right-hand side" in the commutation rules (2). We dare say that this apparently minor mathematical property is perhaps the most important physical difference between Galilei and Poincaré invariance, explaining why it is so easy to construct nontrivial galilean theories, whereas this proves a formidable task in the relativistic case. Suppose indeed that, in the nonrelativistic case first, we start by writing down the explicit representation of the Lie algebra (2) for a world with free, non-interacting, particles. This is a standard matter. We want now to introduce some interaction between the particles, in the simplest possible way. We try, for instance, to add to the free Hamiltonian $H_{0}$, an interaction term $H_{I}$. Looking at the commutation rules (2), it is seen that provided $H_{I}$ is completely invariant under Galilean transformations, i.e. commutes with $(\mathbf{P}, \mathbf{J}, \mathbf{K}, M)$, these rules are not modified when $H_{0}$ is replaced by $H=H_{0}+H_{I}$. Since it is easy, as will be shown, to construct such interaction terms $H_{I}$, we have a very simple way of constructing theories with interacting particles. As an obvious consequence, note that any constant can be added to the Hamiltonian without modifying the equivalence class of the representation. This situation is to be compared to the relativistic one, where any modification of the Hamiltonian, since it appears in the commutator of space-translations and pure Lorentz transformations generators, requires a subsequent modification of other elements in the Lie algebra. The whole structure of the Poincare group representation has thus to be deeply changed and this proves to be rather difficult to do in an explicit way when other conditions, related to locality, energy spectrum constraints, etc., are to be met simultaneously. Clearly these remarks apply as well to the case of so-called "relativistic particle theories" (no fields) where they can be seen to give rise to even stronger constraints, preventing the existence of very general types of such theories, as has been shown by Eksters [15], while allowing galilean theories with interacting particles.

Postulate 3 (mass and energy spectrum, vacuum). Before stating this postulate, some comments are in order. Let us observe that in relativistic theories, one makes at this stage some assumptions about the energy spectrum of the system as given by the Lorentz-invariant squared mass operator $P_{\mu} P^{\mu}[7]$. In the galilean case, the analogous invariant of the Galilei group Lie algebra is the operator [12],[14]:

$$
Q_{1}=2 M H-\mathbf{P}^{2} \stackrel{\text { def }}{=} 2 M W
$$

where we have defined the "internal energy" operator $W$. But nothing 12 Commun. Math. Phys., Vol. 4 
very stringent can be said a priori about the spectrum of $Q_{1}$ (or $W$ ). Indeed, we already pointed out that in each dynamically independent subspace of $\mathscr{H}$, the energy can be modified by an arbitrary amount without any physical consequences [14], as is already the case in classical nonrelativistic physics. The most we can require is that in each eigenspace of the mass operator, there is a lower bound to the internal energy of the system.

As to the mass spectrum proper, i.e. the spectrum of $M$, one could think of avoiding negative eigenvalues. However, particles with opposite mass-eigenvalues can be interpreted as a particle-antiparticle couple [14] so that we would like to preserve the possibility of describing pair production processes and consequent vacuum polarization. The only restriction on the mass spectrum comes from the fact that we want a vacuum state to exist. If we insist that the vacuum is non-degenerate, its invariance under galilean transformations requires it to be a zeromass state, with arbitrary but sharp internal energy and zero momentum. It would be interesting to consider the possibility of a degenerate vacuum in connection with the problem of spontaneous breakdown of symmetries and Goldstone theorem in this nonrelativistic situation; we shall, however, disregard this possibility here. The mass spectrum, in the simplest examples of galilean quantum field theories, will be discrete, but there is no difficulty in considering continuous mass spectra, provided the origin is an isolated point, in order to avoid infrared divergences. Moreover, although zero-mass particles which can be defined in a galilean context [14], may be included, we will not consider this possibility here either. Our postulate then reads:

The mass spectrum contains the origin as an isolated point. There is only one normalizable zero-mass state, the vacuum, which is invariant under galilean transformations (up to a possible phase factor accounting for a constant energy). In each mass eigenspace, the internal energy spectrum is bounded from below.

Notice that if particles and antiparticles, with masses $(m)$ and $(-m)$ respectively, are admitted in the theory, zero-mass states other than the vacuum can be formed. However, as in the relativistic case, the vacuum is the only translation-invariant normalizable state.

Let us also remark that, according to what has been said on the mass conservation law, it is clear that for a theory to allow production processes, the spectrum of the mass operator $M$ must be such that there exists linear relations with integer coefficients between some eigenvalues:

$$
n_{1} m_{1}+n_{2} m_{2}+\cdots=n_{1}^{\prime} m_{1}^{\prime}+n_{2}^{\prime} m_{2}^{\prime}+\cdots
$$

where $n_{i}, n_{i}^{\prime}$ are positive integer numbers and $m_{i}, m_{i}^{\prime}$ eigenvalues of $M$. 
Postulate 4 (Fields). The fundamental dynamical variables of the theory are local fields $\Phi(\mathbf{x}, t)$ associated with a given mass value $m$. They transform locally under a Galilean transformation:

where:

$$
\mathfrak{U}(g)^{-1} \Phi(\mathbf{x}, t) \mathfrak{H}(g)=\exp [i m \gamma(g ; \mathbf{x}, t)] \cdot \Phi\left(\mathbf{x}^{\prime}, t^{\prime}\right)
$$

$$
\begin{gathered}
g=(b, \mathbf{a}, \mathbf{v}, R) \\
\gamma(g ; \mathbf{x}, t)=\frac{1}{2} \mathbf{v}^{2} t+\mathbf{v} \cdot R \mathbf{x}
\end{gathered}
$$

and

$$
\left\{\begin{array}{l}
\mathbf{x}^{\prime}=R \mathbf{x}+\mathbf{v} t+\mathbf{a} \\
t^{\prime}=t+b
\end{array}\right.
$$

This transformation law follows from the analysis of the unitary representations of the Galilei group [11], [13], [14]. The fact that the fields have to carry a definite mass follows from Bargmann's superselection rule which forces the fields to cause transitions from one coherent subspace to another. Another consequence is that no galilean field can be hermitian unless it carries zero mass; mass really here has to be thought of as a special kind of charge. This also implies that the mass of a given field cannot be changed by the introduction of interactions and thus will not have to be renormalized. The transformation law (5) corresponds to the case of spin-zero fields. Spin is a perfectly consistent galilean concept $[12],[14],[16]$, associated to the invariant

$$
Q_{2}=(M \mathbf{J}-\mathbf{K} \times \mathbf{P})^{2}=M^{2} \mathbf{S}^{2}
$$

of the Galilei group Lie algebra. There is thus no difficulty in considering fields with higher spins, obeying transformation laws of the form

$$
\mathfrak{U}(g)^{-1} \Phi_{\sigma}(\mathbf{x}, t) \mathfrak{U}(g)=\exp [i m \gamma(g ; \mathbf{x}, t)] \cdot \sum_{\sigma^{\prime}} \Delta_{\sigma \sigma^{\prime}}(g) \Phi_{\sigma^{\prime}}\left(\mathbf{x}^{\prime}, t^{\prime}\right)
$$

with the same notation as in (5), and relevant spin rotation matrices $\Delta$ [16]. Needless to say, the fields $\Phi(\mathbf{x}, t)$ should be considered as operatorvalued distribution [7].

Free fields are easily defined as creating or annihilating free particles, i.e. particles characterized by an irreducible representation of the Galilei group; besides precise mass and spin values, these particles have well defined internal energies [12], [14], so that their energy $E$ and momentum $\mathbf{p}$ obey the relationship:

$$
E-\frac{\mathbf{p}^{2}}{2 m}=U=\text { const . }
$$

The Fourier transform of the configuration-space field, i.e. the momentumspace field, then has a support restricted to this ,internal-energy shell" (10). Parametrizing this paraboloid (10) by the three-momentum p, we 
can write the considered free field as:

$$
\Phi(\mathbf{x}, t)=(2 \pi)^{-3 / 2} \int d^{3} p \exp [-i E t+i \mathbf{p} \cdot \mathbf{x}] A(\mathbf{p})
$$

where relation (10) is understood to hold. Conversely:

$$
A(\mathbf{p})=(2 \pi)^{-3 / 2} \int d^{3} x \exp [i E t-i \mathbf{p} \cdot \mathbf{x}] \Phi(\mathbf{x}, t) .
$$

The transformation properties of the momentum-space free field, deduced from the configuration space properties (5) by means of (12) are:

$$
\mathfrak{U}(g)^{-1} A(\mathbf{p}) \mathfrak{U}(g)=\exp \left(-i E^{\prime} b+i \mathbf{p}^{\prime} \cdot \mathbf{a}\right) \cdot A\left(\mathbf{p}^{\prime}\right)
$$

with the notations of $(6)$ and:

$$
\left\{\begin{aligned}
\mathbf{p}^{\prime} & =R \mathbf{p}+m \mathbf{v} \\
E^{\prime} & =\frac{\mathbf{p}^{\prime 2}}{2 m}+U \\
& =E+\mathbf{v} \cdot R \mathbf{p}+\frac{1}{2} m \mathbf{v}^{2}
\end{aligned}\right.
$$

Postulate 5 (Local commutativity). At equal times, two field operators either commute or anti-commute (for non-zero spatial separation).

This condition will be taken to mean, as usual, that we want simultaneous "measurements" to be independent. As in the relativistic case [7] of course, this is the least transparent of the postulates, but we do not wish to dwell on this point.

The equal-time situation is just what is left of the space-like separation situation of the relativistic case, in the galilean limit where the invariant light cone is flattened down on the time = const. hyperplane.

It is at this point that we encounter one of the most significant differences between relativistic and galilean quantum field theories. Indeed, in the relativistic case, local commutativity, requiring the fields to commute or anticommute for any space-like separation, is a most powerful condition. It is seen here that the corresponding galilean statement bears only on a space-time region of lesser dimensionality and is accordingly reduced to the status of a rather mild constraint.

As a consequence, the whole structure of the theory loses a great part of its rigidity and some of the most powerful results of relativistic quantum field theory are no longer valid.

This is the case for the CPT theorem, as well as for the Spin-Statistics theorem, the proof of which depends in a crucial way on relativistic local commutativity [7]. It is thus seen how the present considerations may serve a contrario to emphasize the highly non-trivial nature and deep significance of these results obtained in general relativistic quantum field theory. Conversely, since we do not pretend to explain high-energy physics by galilean considerations, but only to construct some field theory models, we will be able to take advantage of these losses. More precisely, we see that there is no obligation in a galilean quantum field theory to treat particles and antiparticles on the same footing; in 
other words, crossing properties are not required. Similarly, perfectly consistent theories can be built with, for instance, spin-zero fermions.

It is instructive to illustrate these remarks in the free field case. Let $A(\mathbf{p})$ and $B(\mathbf{p})$ respectively be momentum-space field operators for a free, spin-zero, particle with mass $m$ and its antiparticle, with mass $(-m)$. They obey canonical commutation or anticommutation relations:

$$
\left[A(\mathbf{p}), A^{*}\left(p^{\prime}\right)\right]_{ \pm}=\left[B(\mathbf{p}), B^{*}\left(\mathbf{p}^{\prime}\right)\right]_{ \pm}=\delta^{(3)}\left(\mathbf{p}-\mathbf{p}^{\prime}\right)
$$

and transform under galilean transformation as indicated in (13)-(14), with the appropriate mass. It is now easy to construct a configurationspace field operator $\Phi(\mathbf{x}, t)$ with the correct transformation properties (5), by Fourier transforming a superposition of particle creation and antiparticle annihilation operators:

$$
\begin{aligned}
\Phi(\mathbf{x}, t)=(2 \pi)^{-3 / 2} \int d^{3} p[\xi \exp (-i E t & +i \mathbf{p} \cdot \mathbf{x}) A(p)+ \\
& \left.+\eta \exp (i E t-i \mathbf{p} \cdot \mathbf{x}) B^{*}(\mathbf{p})\right]
\end{aligned}
$$

where $E=\frac{\mathbf{p}^{2}}{2 m}$, and $\xi, \eta$ are, up to now, arbitrary complex numbers. Computing the commutation relations of this field, we obtain, for equal times:

$$
\left[\Phi(\mathbf{x}, t), \Phi^{*}(\mathbf{y}, t)\right]_{ \pm}=\left(|\xi|^{2} \pm|\eta|^{2}\right) \delta^{3}(\mathbf{x}-\mathbf{y})
$$

so that local commutativity is satisfied for any value of $\xi$ and $\eta$, and in particular for $\eta=0$, corresponding to the absence of antiparticles. Similarly, local commutativity is obeyed for both commutation and anticommutation rules ${ }^{5}$. In other words neither the TCP theorem, nor the spin-statistics connection have to be obeyed, at least in this free-field theory. This situation is to be contrasted with the relativistic case where the requirements of local commutativity on a free field constructed as in (16) impose both the existence of a TCP operation (corresponding to the equal contribution of particles and antiparticles, i.e. $|\xi|=|\eta|$ ), and the spin-statistics relation, as has been shown in a very illuminating way, for this free-field case, by WEINBERG [17].

Perhaps still more important than the failure of TCP and spinstatistics theorems, is the breakdown of Haag's theorem, also implied by the considerable weakening of the local commutativity condition in galilean quantum field theory. Haag's theorem [7], which, loosely speaking, asserts the inexistence of the interaction representation, explains the great difficulty met in trying to build consistent nontrivial

5 It is curious to notice that for an equal admixture of particle and antiparticle in the field (16), i.e. $|\xi|=|\eta|$, the commutator (17) identically vanishes. More generally, for fields with such a crossing symmetry, the equal-time commutator (resp. anticommutator) identically vanishes for integer (resp. half-odd-integer) spin. This property can be viewed as the combined galilean shadow of the CPT and spinstatistics theorems of the relativistic case. Its physical significance, if any, is unknown to us. 
relativistic quantum field theories, by showing the huge qualitative difference between trivial and non-trivial theories. The fact that it does not hold in a galilean quantum field theory will be shown by obvious counterexamples. Incidentally, the breakdown of Haag's theorem in the present situation, that is in genuine field theories, shows that it is not only the introduction of an infinite number of degrees of freedom which brings about this theorem, but also the very compelling requirements associated with relativistic invariance.

Finally, let us mention that the failure of all these powerful results in the present case occurs at the very beginning of the usual abstract approach to relativistic quantum field theory. Indeed, due to the weakening of the spectral condition and local commutativity, it is seen that the Wightman functions of a galilean field theory (vacuum expectation values of products of field operators) lack the very rich and precise analytic properties which make them so useful a tool [7]. For instance, some non-trivial theories, as those considered in the next sections, have two-point Wightman functions identical to those of the free-field case [2], which is not possible in relativistic quantum field theories (Jost's theorem) [7].

Further postulates (Irreducibility, asymptotic condition, etc.). We refrain from formulating additional necessary or desirable properties of the theory since it is not our purpose to develop a complete axiomatic framework for galilean quantum field theory, but rather to emphasize the distinguishing features of such a theory in comparison to relativistic theories and to discuss some specific examples.

\section{Simple examples and counter-examples}

a) Second-quantized nonrelativistic quantum mechanics. The simplest example of a consistent nontrivial galilean field theory, as already pointed out, is well known: we simply have in mind the so-called "secondquantized" form of the Schrödinger equation, describing systems with arbitrary number of particles interacting via two-body forces. The preceding discussion applies in almost a trivial manner. Let us however briefly discuss the conditions galilean invariance imposes on the possible interactions. We consider a spinless field with mass $m, \Phi(\mathbf{x}, t)$, whose transformation properties under galilean transformations have been described in (5-7). In conformity with the discussion of the last section, we write the total Hamiltonian

$$
H=H_{0}+H_{I}
$$

where $H_{0}$ is the free Hamiltonian:

$$
H_{0}=\int d^{3} x \Phi^{*}(\mathbf{x}, t)\left(-\frac{\Delta}{2 m}\right) \Phi(\mathbf{x}, t)
$$


and $H_{I}$ an interaction Hamiltonian describing the two-body force:

$H_{I}=\int d^{3} x d^{3} x^{\prime} d^{3} y d^{3} y^{\prime} \Phi^{*}(\mathbf{x}, t) \Phi^{*}(\mathbf{y}, t) \Phi\left(\mathbf{x}^{\prime}, t\right) \Phi\left(\mathbf{y}^{\prime}, t\right) F\left(\mathbf{x}, \mathbf{x}^{\prime}, \mathbf{y}, \mathbf{y}^{\prime}\right)$.

Requiring $H_{I}$ to be invariant under galilean transformations by using the transformation properties $(5-7)$ of the field, conditions are found on the function $F$ such that the most general permissible interaction Hamiltonian takes the form:

$$
\begin{aligned}
& H_{I}=\int d^{3} z d^{3} x d^{3} y \Phi^{*}\left(\mathbf{z}+\frac{\mathbf{x}}{\mathbf{2}}, t\right) \Phi^{*}\left(\mathbf{z}-\frac{\mathbf{x}}{2}, t\right) \\
& \Phi\left(\mathbf{z}+\frac{\mathbf{y}}{\mathbf{2}}, t\right) \Phi\left(\mathbf{z}-\frac{\mathbf{y}}{\mathbf{2}}, t\right) V(|\mathbf{x}|,|\mathbf{y}|,|\mathbf{x}-\mathbf{y}|)
\end{aligned}
$$

It is not hard to show that this is exactly the second-quantized version of a theory where the two-body interaction is characterized by the nonlocal (in general) potential $V$, entering the Schrödinger equation for the relative motion of two particles. For additional studies of this case and the asymptotic condition in particular, see references [3], [4].

Let us only point out to the use of Fock space as the Hilbert space of the theory, which exemplifies the failure of Haag's theorem.

We note, however, that these theories do not permit any production process. Incidentally, the existence of such non-trivial scattering theories without production processes also is a radical departure from the situation in relativistic quantum field theories, related to the absence of crossing symmetry.

A simple formal modification of this theory can lead however to a theory with creation and annihilation processes. Consider two fields $\Phi$ and $\Psi$ with the same mass $m$, associated to particles which we call $A$ and $B$. Then to an interaction Hamiltonian describing pure scattering processes $A+A \leftrightarrow A+A, B+B \leftrightarrow B+B$ and $A+B \leftrightarrow A+B$, can be superposed a term:

$$
\begin{aligned}
H_{I}^{\prime}= & \int d^{3} z d^{3} x d^{3} y\left[\Phi^{*}\left(\mathrm{z}+\frac{\mathbf{x}}{2}, t\right) \Phi^{*}\left(\mathrm{z}-\frac{\mathbf{x}}{2}, t\right) \times\right. \\
& \left.\times \Psi\left(\mathrm{z}+\frac{\mathbf{y}}{2}, t\right) \Psi\left(\mathrm{z}-\frac{\mathbf{y}}{2}, t\right) W(\mathbf{x}, \mathbf{y})+h . c .\right]
\end{aligned}
$$

which describes pair annihilation and production processes $A+A \leftrightarrow$ $\leftrightarrow B+B$.

b) The polaron model. The polaron model [18], [19] is a simple model describing electron-lattice interactions in polar crystals. It is maybe the simplest example of a really nontrivial quantum field theory and has a great interest, from the practical as well as from the theoretical point of view. Since it describes an obviously nonrelativistic situation, we may wonder if this model is an example of a galilean quantum field theory. The answer is no, although the model describes a galilean invariant system, namely the electron plus the lattice. We have in fact a rather peculiar situation where, so to speak, the dynamical and the 
kinematical degrees of freedom of the system are almost independent. Indeed, at the kinematical level, the electron interacts with the lattice as a whole. For instance, the changes in momentum of the electron are transmitted globally to the (macroscopic) crystal lattice. On the other hand, the electron is dynamically coupled to the phonons (lattice vibrations) which are internal excitations of the lattice. The polarization field of the lattice, of which the phonons are the quanta, thus is not a galilean quantum field, as exhibited by the fact that these phonons do not carry any momentum. As a consequence, there is a well-defined privileged frame of reference for the dynamical problem, namely the crystal rest system, although, let us repeat, the system as a whole is galilean invariant. This analysis is carried out in detail in the review article by FröHLICH [18]. Similar considerations may be applied to almost all the many-body problems where the existence of a macroscopic system defines such a privileged frame of reference, putting the dynamical problem on a purely static basis, and denying any non-trivial consequence to galilean invariance.

c) Static models. There are a number of interesting static models in quantum field theory, describing the interactions between supposedly "light" particles whose energy depends on momentum, and "heavy" particles with fixed energy, whose recoil is neglected: let us quote the neutral scalar model, the Lee model, the Wentzel model, the Van HoveRuijgrok models and the Chew-Low theory [20]. Is it possible to modify these theories in such a way that they become Galilei invariant? A proposal in this direction was made by FeYNMAN [21] for the case of the static meson-nucleon coupling theory. But in general Galilei invariance cannot be achieved in such models because of their incompatibility with the mass conservation law ${ }^{6}$. Indeed, the scalar model, the Van HoveRuijgrok models and the Chew-Low model describe theories with trilinear couplings between fields, say $A_{1}, A_{2}$ and $B$, where both the transitions $A_{1} \leftrightarrow A_{2}+B$ and $A_{2} \leftrightarrow A_{1}+B$ are permitted. Whatever masses are given to the fields, these processes are not both permissible in a galilean theory because of mass conservation, unless the mass of the $B$ field vanishes, case which we do not wish to consider here.

However, the Lee model presents the peculiarity of only allowing one type of transition. This leads to the possibility of recasting it in a galilean invariant manner, as we will presently show.

\section{A "Galilee" model}

a) The Hamiltonian. As in the usual Lee model [22], [23], the theory is defined by an Hamiltonian most simply written in terms of three

\footnotetext{
${ }^{6}$ See in this connection exercise 12, p. 840 of [20].
} 
Schrödinger-picture, momentum-space fields $\mathrm{V}, \mathrm{N}, \theta$ with respective masses $\mathscr{M}, M, m$. To these fields will be associated, in a way to be described, particles with the same respective masses and denoted by $\mathrm{V}, \mathrm{N}, \theta$ as well.

Canonical commutation relations (for the boson field $\theta$ ) and anticommutation relations (for the fermion fields $V$ and $N$ ) are obeyed

$$
\begin{gathered}
\left\{\mathrm{V}(\mathbf{p}), \mathrm{V}^{*}\left(\mathbf{p}^{\prime}\right)\right\}=\left\{\mathrm{N}(\mathbf{p}), \mathrm{N}^{*}\left(\mathbf{p}^{\prime}\right)\right\}=\delta^{(3)}\left(\mathbf{p}-\mathbf{p}^{\prime}\right) \\
{\left[\theta(\mathbf{k}), \theta^{*}\left(\mathbf{k}^{\prime}\right)\right]=\delta^{(3)}\left(\mathbf{k}-\mathbf{k}^{\prime}\right)}
\end{gathered}
$$

all other commutators or anticommutators, depending on the case, vanishing. The Hamiltonian is written as

$$
\begin{aligned}
& H=H_{0}+H_{I} \\
& H_{0}= \int d^{3} P\left(\frac{\mathbf{p}^{2}}{2 \mathscr{M}}+U_{0}\right) \mathrm{V}^{*}(\mathbf{P}) \mathrm{V}(\mathbf{P})+ \\
&+\int d^{3} p \frac{\mathbf{p}^{2}}{2 M} \mathrm{~N}^{*}(\mathbf{p}) \mathrm{N}(\mathbf{p})+\int d^{3} k \frac{\mathbf{k}^{2}}{2 m} \theta^{*}(\mathbf{k}) \theta(\mathbf{k}) \\
& H_{I}= \lambda_{0} \int d^{3} P d^{3} p d^{3} k F(\mathbf{P}, \mathbf{p}, \mathbf{k})\left[\mathrm{V}^{*}(\mathbf{P}) \mathrm{N}(\mathbf{p}) \theta(\mathbf{k})+\theta^{*}(\mathbf{k}) \mathrm{N}^{*}(\mathbf{p}) \mathrm{V}(\mathbf{P})\right]
\end{aligned}
$$
only allowing the fundamental processes $V \leftrightarrow N+\theta$. Notice that we have taken the internal energies associated to the $\mathrm{N}$ and $\theta$ fields to vanish. This can be shown to be no restriction, resulting from the general freedom in the choice of the energy scale origin, in independent subspaces of the Hilbert space. Our first task is to enforce galilean invariance on our theory. The free Hamiltonian $H_{0}$ has the standard form of a free field galilean theory. As shown in the first section, it is sufficient then to insure the interaction Hamiltonian $H_{I}$ to be invariant under any galilean transformation in order to have an explicitly galilean invariant theory, all the generators of the Galilei group except $H$ retaining their free field form. The first condition comes from the mass conservation law and requires the mass of the $V$ field to be the sum of the masses of $\mathrm{N}$ and $\theta$ fields if the coupling is to be allowed. We thus have:

$$
\mathscr{M}=M+m \text {. }
$$

Next, because of translation invariance

$$
F(\mathbf{P}, \mathbf{p}, \mathbf{k})=\delta^{(3)}(\mathbf{P}-\mathbf{p}-\mathbf{k}) F_{1}(\mathbf{p}, \mathbf{k}) .
$$

Invariance under pure galilean transformations now requires $F_{1}$ to be a function only of the galilean invariant relative momentum of the fields $\mathrm{N}$ and $\theta$ :

$$
F_{1}(\mathbf{p}, \mathbf{k})=F_{2}(\mathbf{q}) \quad \text { where } \quad \mathbf{q}=\frac{M \mathbf{k}-m \mathbf{p}}{M+m} .
$$

Finally, rotation invariance demands $F_{2}$ to be a function of $|\boldsymbol{q}|$ only. We now introduce some notations which we will use consistently; $\mathbf{p}$ and $\mathrm{k}$ denoting momenta of fields, or particles, with masses $M$ and $m$ respectively, we will have to use the classical canonical transformation

$$
\mathbf{P}=\mathbf{p}+\mathbf{k} \quad \mathbf{q}=\frac{M \mathbf{k}-m \mathbf{p}}{M+m} .
$$


Let us recall the identity:

$$
\frac{\mathbf{p}^{2}}{2 M}+\frac{\mathbf{k}^{2}}{2 m}=\frac{\mathbf{P}^{2}}{2 \mathscr{M}}+\frac{\mathbf{q}^{2}}{2 \mu} \quad \text { where } \quad \mu \mathscr{M}=m M .
$$

Finally, we will use the notation

$$
\omega=\frac{q^{2}}{2 \mu}
$$

this quantity having the obvious meaning (see (29)) of the center-of-mass, or internal, energy of the considered $(\mathrm{N} \theta)$ system. With these notations, the interaction Hamiltonian reads:

$$
\begin{aligned}
& H_{I}=\lambda_{0} \int d^{3} P d^{3} p d^{3} k \delta^{(3)}(\mathbf{P}-\mathbf{p}-\mathbf{k}) f(\omega)\left[\mathrm{V}^{*}(\mathbf{P}) \mathrm{N}(\mathbf{p}) \theta(\mathbf{k})+\text { h.c. }\right] \\
& =\lambda_{0} \int d^{3} P d^{3} q f(\omega)\left[\mathrm{V}^{*}(\mathbf{P}) \mathrm{N}\left(\frac{M}{\mathscr{M}} \mathbf{P}+\mathbf{q}\right) \theta\left(\frac{m}{\mathscr{M}} \mathbf{P}-\mathbf{q}\right)+\text { h.c. }\right]
\end{aligned}
$$

where we have defined the cut-off function $f(\omega)=F_{2}(|q|)$. Galilean invariance of the theory now is ensured, irrespective of the cut-off function $f(\omega)$. It is interesting to look at the interaction Hamiltonian in configuration space. One obtains:

$$
\begin{aligned}
H_{I}= & \lambda_{0} \int d^{3} R d^{3} r \hat{f}(\mathbf{r}) \times \\
& \times\left[\mathrm{V}^{*}(\mathbf{R}, t) \mathrm{N}\left(\mathbf{R}-\frac{m}{\mathscr{M}} \mathbf{r}, t\right) \theta\left(\mathbf{R}-\frac{M}{\mathscr{M}} \mathbf{r}, t\right)+\text { h.c. }\right]
\end{aligned}
$$

$\hat{f}$ being the Fourier transform of $f$, and with otherwise obvious notations. As usual, the coupling becomes local in the limit where $f=1$ and we will of course be particularly interested in this local, no cut-off limit.

The usual conservation laws of the Lee model:

$$
\left\{\begin{array}{l}
\mathfrak{R}_{1}=\mathfrak{R}_{V}+\mathfrak{R}_{N} \\
\mathfrak{R}_{2}=\mathfrak{R}_{V}+\mathfrak{R}_{0}
\end{array}\right.
$$

are unchanged and allow us to divide the Hilbert space of the system into independent sectors. In the present case they correspond exactly to the eigenspaces of the mass operator ${ }^{7}$ which can be written as:

$$
\mathfrak{M}=\mathscr{M} \mathfrak{R}_{V}+M \mathfrak{R}_{N}+m \mathfrak{R}_{\theta}=M \mathfrak{R}_{1}+m \mathfrak{R}_{2} .
$$

b) Physical V-particle and N-O scattering. The solution of the model in the lowest sectors is standard [20], [22], [23]. The vacuum and the physical $\mathrm{N}$ - and $\theta$-particle states remain unchanged by the introduction of the interaction. If the parameters $U_{0}, \lambda_{0}$ are such that a stable $\mathrm{V}$-particle exists, let $|\mathrm{V}(\mathbf{P})\rangle$ be the state of this particle with momentum P, normalized by:

$$
\left\langle\mathrm{V}(\mathbf{P}) \mid \mathrm{V}\left(\mathbf{P}^{\prime}\right)\right\rangle=\delta^{(3)}\left(\mathbf{P}-\mathbf{P}^{\prime}\right) .
$$

The wave function renormalization constant is then defined as usual by:

$$
\left\langle 0|\mathrm{~V}(\mathbf{P})| \mathrm{V}\left(\mathbf{P}^{\prime}\right)\right\rangle=Z^{1 / 2} \delta^{(3)}\left(\mathbf{P}-\mathbf{P}^{\prime}\right) .
$$

\footnotetext{
${ }^{7}$ We suppose the ratio $m / M$ to be an irrational number.
} 
Due to the invariance properties, the $V$-particle state can be written:

$$
|\mathrm{V}(\mathbf{P})\rangle=\left[Z^{1 / 2} \mathrm{~V}^{*}(\mathbf{P})+\int d^{3} q \varphi(\omega) \mathrm{N}^{*}\left(\frac{M}{\mathscr{M}} \mathbf{P}+\mathbf{q}\right) \theta^{*}\left(\frac{m}{\mathscr{M}} \mathbf{P}-\mathbf{q}\right)\right]|0\rangle
$$

calling $U$ the internal energy of the physical $V$-particle, we obtain from the eigenvalue equation:

$$
\left\{\begin{array}{l}
Z^{1 / 2}\left(U-U_{0}\right)=-\lambda_{0} \int d^{3} q f(\omega) \varphi(\omega) \\
(\omega-U) \varphi(\omega)=-\lambda_{0} Z^{1 / 2} f(\omega)
\end{array}\right.
$$

and the normalization condition (35) gives

$$
Z+\int|\varphi(\omega)|^{2} d^{3} q=1 .
$$

As may be expected, a normalizable state results only if the internal energy eigenvalue is negative. This may be achieved for any value of $\lambda_{0}$ by suitable choice of the "bare" energy $U_{0}$. In the local coupling limit ( $f=1$, no cut-off), $U_{0}$ has to be chosen infinite (negative) to produce a finite $U$ (infinite energy renormalization) but, in contrast to the usual Lee model, $Z^{-1}$ will remain finite for any finite, real $\lambda_{0}$. The renormalization constant is computed as:

$$
Z^{-1}=1+\frac{\lambda_{0}^{2}}{\lambda_{c}^{2}}
$$

where the critical coupling constant is, in the no-cut-off case:

$$
\lambda_{c}^{2}=\frac{1}{\pi^{2}}\left(\frac{-U}{2 \mu^{3}}\right)^{1 / 2} .
$$

One easily checks that for real $\lambda_{0}$, there is no ghost problem and no other single particle state occurs in the $\mathrm{N}-\theta$ sector. Of course one can introduce a ghost by choosing $\lambda_{0}$ to be imaginary, which is equivalent to insist on the renormalized coupling constant $\lambda=Z^{1 / 2} \lambda_{0}$ being greater than the critical value $\lambda_{c}$.

The full expression for the physical $V$-particle propagator is found to be, in the local coupling case:

where

$$
\Delta_{\nabla}(\Omega)=\left(1+\frac{\lambda_{0}^{2}}{\lambda_{c}^{2}}\right)\left[\Omega-2 \frac{\lambda_{0}^{2}}{\lambda_{c}^{2}}(U \Omega)^{1 / 2}-\left(1+2 \frac{\lambda_{0}^{2}}{\lambda_{c}^{2}}\right) U\right]^{-1}
$$

$$
\Omega=E_{V}-\frac{\mathbf{p}^{2}}{2 \mathscr{M}}
$$

is the (invariant) internal energy of the $\mathrm{V}$-particle.

The calculation of the $\mathrm{N}-\theta$ scattering is now straightforward, due to the close connection, typical of the Lee model, between the N- $\theta$ scattering amplitude and the $\mathrm{V}$-propagator

$$
\exp [2 i \delta(\omega)]=\Delta_{V}(\omega) \overline{\Delta_{V}(\omega)^{-1}} \quad \text { or } \quad \operatorname{cotg} \delta=-\frac{\operatorname{Re} \Delta_{V}^{-1}}{\operatorname{Im} \Delta \bar{v}^{-1}} .
$$

It is particularly amusing that the $\mathrm{N}-\theta$ scattering phase shift, in the case of no cut-off, is given by an exact effective range formula:

$$
q \operatorname{cotg} \delta=-\frac{1}{a}+\frac{1}{2} r_{0} q^{2}
$$


where

$$
\left\{\begin{array}{l}
a=(-2 \mu U)^{-1 / 2} \frac{-2 \lambda_{0}^{2} / \lambda_{c}^{2}}{1+2 \lambda_{0}^{2} / \lambda_{c}^{2}} \\
r_{0}=(-2 \mu U)^{-1 / 2} \frac{1}{\lambda_{0}^{2} / \lambda_{c}^{2}}
\end{array} .\right.
$$

It is seen on these expressions that the scattering length and, more generally, the cross-section at any fixed energy, do not increase indefinitely with the bare coupling constant, contrarily to what could be an intuitive expectation. Instead, the cross-section has a finite upper bound, reached for an infinite value of the bare coupling constant.

The given Hamiltonian (24/31) thus cannot describe scattering processes with a cross-section higher than this maximal value. Only if we ignore this natural bound, does the ghost appear and the model lose its internal consistency. One can wish this to be the case also in more realistic quantum field theories.

c) Elementarity of the $\mathrm{V}$ particle. An interesting question now is the one of the $\mathrm{V}$ particle elementarity. As in the usual case [24], it can be shown that only if $Z=0$, can the same physical results in the $\mathrm{N}-\theta$ sector (i.e. position of the bound state and scattering amplitudes) be obtained from a Hamiltonian without any $\mathrm{V}$ field and where the particles $\mathrm{N}$ and $\theta$ interact via a separable potential

$$
V(\mathbf{x}, \mathbf{y})=\hat{f}(\mathbf{x}) \hat{f}(\mathbf{y})
$$

$\hat{f}$ being the Fourier transform of the cut-off function $f$. These results are confirmed by the use of Levinson's theorem. Indeed from the general expression for the phase-shift, with $f \neq 1$ for the time being, it can be seen that:

if $Z \neq 0$, then $\delta(0)-\delta(\infty)=0$ (no bound state),

if $Z=0$, then $\delta(0)-\delta(\infty)=\pi$ (one bound state).

In the local case, the condition $Z=0$ also is equivalent to the nonelementarity of the V-particle which can now appear as the bound state of the $\mathrm{N}$ and $\theta$ particles interacting via a contact interaction (or equivalently, a so-called " $\delta$-potential"). However in this case, Levinson's theorem does not apply since one finds (see $(45,46))$ :

if $Z=0$, then in the local limit $\delta(0)-\delta(\infty)=\frac{\pi}{2}$.

This appearance of a "half-bound" V-particle obviously is due to the singularity of the "potential". But it has the interest of being a counterexample to Levinson's theorem in field theory. Indeed, in order to prove the theorem [25], one requires the imaginary part of the propagator to be of order unity as the energy increases indefinitely. This assumption is seen not to be satisfied by the local-coupling propagator (43).

d) The case of an unstable V-particle. To conclude our investigation of the $\mathrm{N}-\theta$ sector, it is of interest to treat the case of an unstable particle 
[26], [27], [28], since the existence of a meaningful local-coupling limit gives simple explicit results. Notice that, contrarily to our assertion in [14], the mass superselection rule does not prevent the existence of galilean unstable particles. These have a sharp mass value but an internal energy spectrum whose width is related to the lifetime. We start from the unrenormalized Hamiltonian (24/31) with $U_{0}>0$ now.

The V-particle propagator is:

$$
\Delta_{V}(\Omega)=\left[\Omega-U_{0}-\lambda_{0}^{2} \int d^{3} q \frac{|f(\omega)|^{2}}{\Omega-\omega+i \varepsilon}\right]^{-1} .
$$

The same expressions (45) as in the stable case hold for the N- $\theta$ scattering phase-shift. We can first define the internal energy of the V-particle by the position of the resonance i.e. by the condition:

$$
\delta(U)=\frac{\pi}{2} \quad \text { or } \operatorname{Re} \Delta_{V}(U)^{-1}=0 .
$$

This gives the relation:

$$
U-U_{0}-\lambda_{0}^{2} \mathscr{P} \int d^{3} q \frac{|f(\omega)|^{2}}{U-\omega}=0
$$

between the renormalized and unrenormalized energies. In terms of the renormalized quantity, the propagator reads:

$$
\Delta_{V}(\Omega)=(\Omega-U)^{-1}\left[1-\lambda_{0}^{2} \int d^{3} q \frac{|f(\omega)|^{2}}{(U-\omega)(\Omega-\omega+i \varepsilon)}\right]^{-1} .
$$

A reasonable definition of the renormalization constant is obtained by renormalizing the propagator:

$$
\Delta_{V}^{R}=Z^{-1} \Delta_{V}
$$

so that the coefficient of the pole term $(\Omega-U)$ in $\operatorname{Re} \Delta_{V}^{R-1}$ be unity that is:

which gives

$$
Z^{-1}=\left.\frac{\partial}{\partial \Omega} \operatorname{Re} \Delta_{V}(\Omega)^{-1}\right|_{\Omega=U}
$$

$$
Z^{-1}=1-\lambda_{0}^{2} \mathscr{P} \int d^{3} q \frac{|f(\omega)|^{2}}{U-\omega},
$$

The width $\gamma$ of the $V$-particle is now defined by the slope of the phaseshift function at resonance, writing:

This gives:

$$
\operatorname{cotg} \delta \cong-\frac{\omega-U}{\gamma / 2} \text { for } \omega \cong U
$$

$$
\frac{1}{2} \gamma=\operatorname{Im} \Delta_{V}^{R}(U)^{-1}=Z 4 \pi^{2} \lambda_{0}^{2} \mu q_{U}|f(U)|^{2}
$$

where $q_{U}$ is the relative momentum at resonance $\left(U=\frac{q_{U}^{2}}{2 \mu}\right)$. The no cut-off case turns out to be particularly simple since, when computing the relevant integral, one finds:

$$
\operatorname{Re} \Delta_{V}(\Omega)^{-1}=\Omega-U
$$

and, in particular, $Z=1$ and $\Delta_{V}^{R}=\Delta_{V}$. 
The phase-shift is then given by:

where

$$
\operatorname{cotg} \delta=-\frac{\omega-U}{\gamma / 2} \frac{q_{0}}{q}
$$

$$
\frac{1}{2} \gamma=4 \pi^{2} \lambda_{0}^{2} \mu q_{U}
$$

so that the total cross-section reads:

$$
\sigma(\omega)=\frac{4 \pi}{2 \mu U} \frac{(\gamma / 2)^{2}}{(\omega-U)^{2}+\left(\frac{\gamma}{2}\right)^{2} \frac{\omega}{U}},
$$

Amusingly enough, this can be written in an exact Breit-Wigner form:

by defining:

$$
\sigma(\omega)=\sigma_{0} \frac{\left(\gamma^{\prime} / 2\right)^{2}}{\left(\omega-U^{\prime}\right)^{2}+\left(\gamma^{\prime} / 2\right)^{2}}
$$

$$
\left\{\begin{array}{l}
U^{\prime}=U\left(1-\frac{1}{8} \frac{\gamma^{2}}{U^{2}}\right) \\
\gamma^{\prime 2}=\gamma^{2}\left(1-\frac{1}{16} \frac{\gamma^{2}}{U^{2}}\right)
\end{array}\right.
$$

More generally, it is seen that $\left(U^{\prime}, \gamma^{\prime}\right)$ may be defined by the position of the pole of the propagator (in the second sheet):

$$
\left[\Delta_{V}\left(U^{\prime}-i \frac{\gamma^{\prime}}{2}\right)\right]^{-1}=0
$$

Since, in this no cut-off case, we have there:

$$
\Delta_{V}(\Omega)=\left[\Omega-U+i \frac{\gamma}{2}\left(\frac{\Omega}{U}\right)^{1 / 2}\right]^{-1}
$$

we recover the expressions (63).

Due to the existence of a well-defined local coupling limit, we thus see how our model provides a particularly simple example for comparing the various possible definitions for the position and width of a resonance, without necessitating complicated discussions on the analytic behavior of functions depending upon the arbitrary cut-off function [28].

e) Conclusions. Let us now summarize our results on this modified Lee model ${ }^{8}$. Firstly, we have shown the existence of non-trivial galilean quantum field theories exhibiting production processes, renormalization procedures, etc., i.e. physical effects typical of a true quantum field theory.

Secondly, the model as such has the obvious advantage over the original Lee model of giving meaningful results for the case of a local

${ }^{8}$ Similar calculations have been performed by WeLLNER [29] and SANDRI (see especially footnote 3 of WeLLNER's article). WELLNER has pointed out the close relationship between the Wigner-Weisskopf model and the $\mathrm{V} \leftrightarrow \mathrm{N}-\theta$ sector of a galilean Lee model. I thank Dr. WeLLNER for bringing his paper to my attention. 
coupling, instead of leading to the ghost difficulties. Of course, the reason for these modifications is not very profound and, grossly speaking, results from the replacement of relativistic energy denominators $\omega=\left(\mu^{2}+q^{2}\right)^{1 / 2}$ by nonrelativistic ones $\omega=q^{2} / 2 \mu$, thereby greatly improving the convergence properties of the various integrals expressing the physical results ${ }^{9}$.

What we would like to argue is that this modification does not decrease the interest of the Lee model. Indeed, the usual formulation is kinematically completely inconsistent, since two particles are treated statically, while the third one is endowed with a relativistic energymomentum relationship. But a still deeper violation of relativistic invariance lies at the very root of the model, which is designed in such a way as to avoid any vacuum polarization, by decoupling the antiparticles. This gives rise, as is well-known, to a model without TCP nor locality and which cannot, accordingly, pretend to simulate any of the effects crucially depending on relativistic invariance in relativistic quantum field theories. The hope to mimic relativistic properties just by modifying the energy-momentum relationship of the $\theta$ particle, thus seems to be rather futile ${ }^{10}$. We think it preferable to recognize at the outset this fundamentally nonrelativistic nature of the model and to try to put it on a sound galilean kinematical basis, which is at least consistent from the nonrelativistic point of view. As we hope to have shown, none of the desirable features of the model are lost, nor are the physical effects modified, while at least some pleasant new properties emerge.

As a conclusion, let us mention the possibility of considering many other such galilean theories. One may for instance adapt easily our considerations to various extended Lee models which have been proposed [28], [35], [36], [37], [38]. A more interesting possibility consists in investigating theories whose fundamental fields have a rather complicated mass spectrum (such as a continuous one, or one consisting of integer multiples of a common mass, etc.) in which the possibilities of production processes are greatly enriched by, so to speak, releasing the frozen degrees of freedom associated with the mass.

It is a pleasure to thank Prof. R. E. Marshak for his hospitality at the University of Rochester where I have benefitted from interesting discussions with Dr. G. Guralnik, Dr. C. Hagen and Prof. H. M. Nussenszveig. Stimulating conversations with Prof. H. Ekstein and Prof. A. S. Wightman also are gratefully acknowledged.

9 These kinematical changes also account for the non-applicability of the LSZ theorem on the vertex functions [30], which explains the appearance of a ghost in the usual Lee model.

10 Further attempts to construct relativistic Lee models [31], [32], [33], [34] may be criticized on the same grounds. 


\section{References}

[1] Ter-Martirosyan, K. A.: Report at the XV. Nuclear Spectroscopy Conference, Tbilisi (1964) (to be published) (review article).

[2] Dresden, M., and P. B. KaHn: Rev. Mod. Phys. 34, 401 (1962).

[3] Redmond, P. J., and J. L. Uretzky: Ann. Phys. (N.Y.) 9, 106 (1960).

[4] Hepp, K.: Lectures at the Brandeis Summer School (1965).

[5] Schweber, S. S.: Ann. Phys. (N.Y.) 20, 61 (1962).

[6] Kramer, G., and K. Meetz: Commun. Math. Phys. 3, 29 (1966).

[7] Streater, R. F., and A. S. Wightman : PCT, spin and statistics and all that. New York: Benjamin 1964.

[8] Wick, G. C., E. P. Wigner, and A. S. Wightman: Phys. Rev. 88, 101 (1952).

[9] Wigner, E. P.: Ann. Math. 40, 149 (1939).

[10] Inönv, E., and E. P. Wigner: Nuovo Cimento 9, 705 (1952).

[11] Bargmann, V.: Ann. Math. 59, 1 (1954), especially sections 6f, $6 \mathrm{~g}$.

[12] Hamermesh, M.: Ann. Phys. (N.Y.) 9, 518 (1960).

[13] Wightman, A. S.: Rev. Mod. Phys. 34, 845 (1962), especially section 6 .

[14] Lévy-Leblond, J.-M.: J. Math. Phys. 4, 776 (1963).

[15] Ekstein, H.: Commun. Math. Phys. 1, 6 (1965).

[16] Lévy-Leblond, J.-M.: Orsay preprint TH/112, November 1965 (to be published).

[17] Weinberg, S.: Phys. Rev. 133, B 1318 (1964).

[18] Fröhlich, H.: Adv. Phys. 3, 325 (1954) and additional references therein.

[19] Höhlen, G.: In: Field theory and the many-body problem, E. R. CaIAniello, editor, p. 285 and additional references therein. New York: Academic Press 1959.

[20] SCHWEBER, S. S.: Introduction to relativistic quantum field theory. Chapter 12 and original references therein. Evanston: Row and Peterson 1961.

[21] Fermman, R. P.: Proceedings of the Third Annual Conference on High Energy Nuclear Physics, p. 87. Rochester 1952, University of Rochester publ., 1953.

[22] LeE, T. D.: Phys. Rev. 95, 1329 (1954).

[23] Källen, G., and W. PaUli: Kgl. Danske Vidensk. Selsk. Mat.-Fys. Medd. 30, no. 7 (1955).

[24] Houard, J. C., and B. Jouvet: Nuovo Cimento 18, 466 (1960).

[25] Hagen, C. R.: Nuovo Cimento 43, A 596 (1966).

[26] Glaser, V., and G. Källen: Nucl. Phys. 2, 706 (1956/57).

[27] Araki, H., Y. Munakata, M. Kawaguchi, and T. Goto: Progr. Theor. Phys. 17,419 (1957).

[28] LÉvy, M.: Nuovo Cimento 13, 114 (1959) and 14, 613 (1959).

[29] Wellner, M.: Phys. Rev. 118, 878 (1960).

[30] Lehmann, H., K. Symanzik, and W. Zimmermann: Nuovo Cimento 2, 425 (1955).

[31] Gunther, M.: Phys. Rev. 125, 1061 (1962) and 126, 2261 (1962).

[32] Fried, D. L., and L. Sartori: Phys. Rev. 128, 2879 (1962).

[33] Martin, J. L.: Proc. Roy. Soc. (Lond.) A 271, 332 (1963) and A 272, 231 (1963).

[34] Yndurátn, F. J.: J. Math. Phys. 7, 1133 (1966).

[35] Goldstein, J. S.: Nuovo Cimento 9, 504 (1958).

[36] Braun, M. A.: Soviet Physics JETP 18, 647 (1964).

[37] Marr, R. B., and Y. Shimamoto: Nuovo Cimento 31, 599 (1964).

[38] Srivastava, P. K.: Phys. Rev. 126, 2908 (1960). 